\title{
Mid Term Results of Fenestrated Intra-Extracardiac Fontan Procedure: Insights, Experiences and Expectations
}

\author{
Arda Ozyuksel ${ }^{1}$, Baran Simsek $^{1}$, Sener Demiroluk ${ }^{1}$, Murat Saygi $^{1}$, and Mehmet Bilal ${ }^{1}$ \\ ${ }^{1}$ Medicana International Istanbul Hospital
}

November 29, 2021

\begin{abstract}
Background: Intraextracardiac Fontan procedure aimed to combine the advantages of lateral tunnel and extracardiac conduit modifications of the original technique. Herein, we present our experience in our patients with intraextracardiac fenestrated Fontan Procedure. Methods: A retrospective analysis was performed in order to evaluate intraextracardiac fenestrated Fontan patients between 2014 and 2021. Seventeen patients were operated on with a mean age and body weight of $9.1 \pm 5.5$ years and $28.6 \pm 14.6 \mathrm{~kg}$. Results: Sixteen patients $(94 \%)$ were palliated as univentricular physiology with hypoplasia of one of the ventricles. One patient $(6 \%)$ with well-developed two ventricles with double outlet right ventricle and complete atrioventricular septal defect had straddling of the chordae prohibiting a biventricular repair. All of the patients had cavopulmonary anastomosis prior to Fontan completion, except one case. Fenestration was performed in all cases. Postoperative mean pulmonary artery pressures and arterial oxygen saturation levels at follow up were $10 \pm 2.4 \mathrm{mmHg}$ and $91.3 \pm 2.7 \%$, respectively. Mean duration of pleural drainage was $5.4 \pm 2.3$ days. All of the fenestrations are patent at a mean follow up period of $4.8 \pm 7.7$ years, except one case. Any morbidity and mortality were not encountered. Conclusions: The mid-term results of intraextracardiac fenestrated Fontan procedure are encouraging. This procedure may improve the results in a patient population who should be palliated as univentricular physiology, especially in cases with complex cardiac anatomy.
\end{abstract}

\section{Mid Term Results of Fenestrated Intra-Extracardiac Fontan Procedure:}

Insights, Experiences and Expectations

Baran Şimşek ${ }^{1}$, MD; Arda Özyüksel ${ }^{1,2 *}$, MD, Prof.; Şener Demiroluk ${ }^{3}$, MD, Assoc. Prof.; Murat Sayg ${ }^{4}$, MD; Mehmet Salih Bilal ${ }^{1}$, MD, Prof.

${ }^{1}$ Department of Cardiovascular Surgery, Medicana International Hospital, Istanbul, Turkey

${ }^{2}$ Department of Cardiovascular Surgery, Biruni University, Istanbul, Turkey

${ }^{3}$ Department of Anesthesiology, Medicana International Hospital, Istanbul, Turkey

${ }^{4}$ Department of Pediatric Cardiology, Medicana International Hospital, Istanbul, Turkey

Running Head: Intra-extracardiac Fenestrated Fontan Procedure Pressure

Funding / Disclosures: None

Abstract word count: 231

Text word count: 4820

Number of tables: 2

Keywords: congenital heart disease, Fontan Procedure, cavopulmonary anastomosis, single ventricle 
*Address for Correspondence:

Arda Özyüksel, MD, Prof.

Department of Cardiovascular Surgery,

Medicana International Hospital, Beylikduzu Cad. No: 3,

Beylikduzu, Istanbul, 34520, Turkey

e-mail:ozyukselarda@yahoo.com

Tel: +90212 8677500, Fax: +902128677672

\section{ABSTRACT}

Background: Intraextracardiac Fontan procedure aimed to combine the advantages of lateral tunnel and extracardiac conduit modifications of the original technique. Herein, we present our experience in our patients with intraextracardiac fenestrated Fontan Procedure.

Methods: A retrospective analysis was performed in order to evaluate intraextracardiac fenestrated Fontan patients between 2014 and 2021. Seventeen patients were operated on with a mean age and body weight of $9.1 \pm 5.5$ years and $28.6 \pm 14.6 \mathrm{~kg}$.

Results: Sixteen patients (94\%) were palliated as univentricular physiology with hypoplasia of one of the ventricles. One patient $(6 \%)$ with well-developed two ventricles with double outlet right ventricle and complete atrioventricular septal defect had straddling of the chordae prohibiting a biventricular repair. All of the patients had cavopulmonary anastomosis prior to Fontan completion, except one case. Fenestration was performed in all cases. Postoperative mean pulmonary artery pressures and arterial oxygen saturation levels at follow up were $10 \pm 2.4 \mathrm{mmHg}$ and $91.3 \pm 2.7 \%$, respectively. Mean duration of pleural drainage was $5.4 \pm 2.3$ days. All of the fenestrations are patent at a mean follow up period of $4.8 \pm 7.7$ years, except one case. Any morbidity and mortality were not encountered.

Conclusions: The mid-term results of intraextracardiac fenestrated Fontan procedure are encouraging. This procedure may improve the results in a patient population who should be palliated as univentricular physiology, especially in cases with complex cardiac anatomy.

INTRODUCTION Fontan procedure (FP) has been performed as the common destination treatment for patients with single ventricle physiology, since it was defined in early 1970's. At first, Dr. Francis Fontan defined this surgical procedure by performing an atriopulmonary connection in 3 patients with tricuspid atresia, which was the prototype of true single ventricle morphology (1). Following the first years of FP, many modifications were defined as well as the indications of this palliative procedure were extended. Apart from single ventricle morphology, cases in which a satisfactory septation could not be achieved such as double outlet right ventricle (DORV) with a remote ventricular septal defect (VSD), FP was the procedure of choice (2). Probably, there has been no other palliative surgical procedure other than the Fontan circulation in the historical development of congenital heart diseases that had significant modifications with improved results for decades. Moreover, although FP is a palliative in nature, it is also the obligatory definitive treatment in cases where one half of the venricles is not adequately developed to provide a biventricular cardiac pump.

The necessity for improving the results of FP always delighted the surgeons in order to achieve better long-term results. The major disadvantage of first generation atriopulmonary FP was the exposure of the whole atrial tissue to high systemic venous pressure and this unphysiological status of the right atrium led to significant atrial hypertrophy and dilatation, leading to supraventricular arrhythmias (2). Moreover, this type of FP was unsuitable for patients with mitral atresia, who had a continous left to right shunt at the level of atrial septum (3). The first important modification of FP was the lateral tunnel (LT) technique (1988), which primarily aimed to achieve better hemodynamic results when the energy loss in the enlarged atrium in the original technique was considered $(3,4)$. The atrial septal flap and inner right atrial wall were used in combination in order to direct the blood coming from inferior venae cavae (IVC) to pulmonary vascular 
bed in LT technique. A few years later, before the long-term results of LT technique were not clear yet, Marcelletti et al. introduced the second important modification, the extracardiac conduit (ECC) Fontan technique (1990) (5). This was an IVC to pulmonary artery extracardiac conduit in origin, which aimed to eliminate the disadvantages of LT technique, especially the atrial wall still being exposed to high systemic venous pressure same as it occurs in the original atriopulmonary type FP. It was soon demonstrated in a canine model by Rodefeld and colleagues that the suture line in LT technique was an adequate substrate for the atrial rhythm pathways to result in supraventricular arrhythmias (6).

In the same year with the publication of the ECF technique, in 1990, Dr. Bridges and colleagues from Boston reported the idea of fenestration, which was initially performed in high-risk candidates of Fontan circulation (7). Their criteria in order to identify high risk candidates were mean pulmonary artery pressure (MPAP $>18 \mathrm{mmHg}$ ), ventricular end-diastolic pressure $>12 \mathrm{mmHg}$, atrioventricular (AV) valvar regurgitation, pulmonary artery distortion, pulmonary vascular resistance $>2$ Woods' units, systemic ventricular outflow tract obstruction and complex anatomy (7). Interestingly, they reported the feasibility of transcatheter closure of the fenestration in the same paper. This idea of fenestration led to another vast amount of uncertainty, whether to fenestrate or not. It was agreed that fenestration decreased the postoperative pleural effusion duration and length of hospital stay but it was bringing up this advantage with a cost of systemic desaturation (8). Moreover, maintenance of the fenestration patency was questionable in LT and ECF techniques (4).

Although described earlier in the development history of FP, the intra-extracardiac Fontan procedure with fenestration (IECF) was revisited which aimed to combine the advantages of LT and ECF $(4,9,10)$. At the early times of ECF, transection of IVC at cavoatrial junction and primarily suturing the right atrial stump was thought to be innocent with regard to atrial conduction pathways. This modification improved the feasibility of the Fontan circulation in cases with heterotaxy syndromes, provided with a better hemodynamic result, further decreased the problem of arrhythmia with preserving IVC and right atrial continuity as well as providing an easy and durable fenestration on the conduit.

Herein, we aimed to present our experience in patients whom we performed the intra-extracardiac fenestrated Fontan technique, along with discussing the advantages over the other modifications.

\section{MATERIALS and METHODS}

We performed 17 IECF procedures out of a total of 152 Fontan operations in our congenital cardiac surgery center between 2014 and 2021. Medical records; preoperative, operative and postoperative data of these patients were retrospectively analyzed following the approval of institutional review board (IRB).

Seventeen patients (10 males, 7 females) underwent IECF procedure. Mean age and body weight were $9.1 \pm$ 5.5 years (range: 3 to 22 years) and $28.6 \pm 14.6 \mathrm{~kg}$ (range: 15 to $68 \mathrm{~kg}$ ), respectively. All of the cases except patient no. 12 had univentricular physiology in which one of the right $(n=7)$ or left $(n=9)$ ventricles were dominant. The demographic and operative data of the patients are demonstrated in table- 1 .

\section{RESULTS}

Sixteen patients (94\%) had situs solitus and one patient (6\%) had situs inversus. Sixteen patients (94\%) were palliated as univentricular physiology due to the hypoplasia of one of the ventricles. One patient (pt. no 12) with the diagnosis of DORV, Rastelli type C complete atrioventricular septal defect (CAVSD) and pulmonary artery hypoplasia had well developed left and right ventricles. However, the straddling of the chordae prohibited a biventricular repair. Three patients (17\%) (pt. no 2,8 and 16) had tricuspid atresia (TA) and 2 patients (11\%) had mitral atresia (pt. no 5 and 11). We encountered double inlet ventricles in 5 cases (29\%) (pt. no 3,6,7,13 and 15). Three cases (17\%) (pt. no 4,9 and 17) had CAVSD with unbalanced ventricular anatomy where a univentricular palliation was performed. We did not encounter any case with significant arrhythmia, except patient no.1, who underwent successful ablation of the intraatrial reentry tachycardia at the preoperative period.

Stage-1 palliation was deemed necessary in 10 cases (58\%). These interventions included pulmonary banding (PB) alone (11\%) (pt. no 3 and 10), systemic to pulmonary artery shunts alone (11\%) (pt. no 6 and 11), PB 
with concomitant supramitral ring resection and surgical atrial septectomy (pt. no 5), shunt along with major aortopulmonary collateral (MAPCA) banding (pt. no 12), PB with atrial septectomy (pt. no 13), PB with aortic arch reconstruction and coarctation repair (pt. no 15). Balloon atrial septostomy and patent ductus arteriosus stenting were performed in two cases (pt. no 14 and pt. no 16, respectively) via percutaneous route. In one patient (pt. no12), a second operation for stage-1 palliation was deemed mandatory in order to augment the right pulmonary artery blood flow with patch plasty.

Stage-2 palliation included bidirectional cavopulmonary anastomosis and associated interventions, except for patient no. 12 in whom the cavopulmonary anastomosis was performed along with intraextracardiac Fontan (IECF) procedure at stage 3. Systemic-to-pulmonary artery shunts were closed in two patients (pt. no 6 and 11) in stage 2. Concomitant procedures included patent ductus arteriosus ligation (pt. no 9), tricuspid valve repair with PB and pulmonary artery mobilization (pt. no 11), atrial septectomy and PB (pt. no 14), ventricular septal defect enlargement, subaortic membrane resection and atrial septectomy (pt. no 15) and left pulmonary artery patch augmentation (pt. no 16). In patient no.16, left pulmonary artery was stented and a left modified Blalock-Taussig shunt procedure was performed in another cardiac surgery center between stages 2 and 3; the shunt was occluded at the time of IECF. One patient (pt. no 17) had interrupted inferior vena cava with azygos vein continuity and Kawashima procedure was performed at stage 2. Patients no 1 and 10 had bilateral superior vena cava that were anastomosed to the ipsilateral pulmonary arteries at stage 2 .

Stage-3 palliation included fenestrated IECF completion procedure in all cases. A polytetrafluorethylene (PTFE) tubular conduit (GORE-TEX?, W.L. Gore and Associates Ltd., Livingston, Scotland) with a size of 18 or $20 \mathrm{~mm}$ was used in all cases. A fenestration of $4,4.5$ or $5 \mathrm{~mm}$ was performed with an aortic punch. Concomitant procedures at stage III included atrial septectomy (pt.no 1 and 2), AV valve repair (pt.no 9, 10 and 15) and pulmonary artery reconstruction (pt.no 12 and 15). All of the patients were evaluated with cardiac catheterization before Fontan completion. The mean pulmonary artery (Nakata) index was $209+-$ $67 \mathrm{~mm}^{2} / \mathrm{m}^{2}$ (range: $97-315 \mathrm{~mm}^{2} / \mathrm{m}^{2}$ ). Mean preoperative pulmonary artery pressure that was directly measured at catheterization was $13.8+-3.3 \mathrm{mmHg}$ (range: $8-21 \mathrm{mmHg}$ ), whereas the postoperative MPAP measured at the central venous line was $10+-2.4 \mathrm{mmHg}$ (range: $4-14 \mathrm{mmHg}$ ). The stages of Fontan circulation and concomitant procedures are summarized in table-2.

\section{Surgical Technique}

Re-sternotomy was performed in all cases. The femoral artery and vein were prepared in cases where the mediastinal structures were adhered to the sternum (determined with lateral chest x-ray or computed tomography in the preoperative evaluation), whereas femoral cannulation and initiation of cardiopulmonary bypass before sternal entrance was not necessary in any case. Following aortic and bicaval (or tricaval in cases with a persistent superior venae cavae), aorta was cross clamped and antegrade blood cardioplegia was administered where intermittent doses were repeated at every 15 minutes. Oblique right atriotomy was performed at the lower half of the atrial wall. Pulmonary arteries were divided above the pulmonary valve except for the cases with pulmonary atresia. Pulmonary valves were either resected or squeezed within the polypropylene double row suture line that will close the pulmonary outflow of the ventricle. If necessary, AV valves were repaired at this stage of the operation. The PTFE conduit with proper size was anastomosed to the orifice of IVC in a beveled end-to-end fashion, carefully observing any accessory hepatic venous drainage to the right atrium. In patients no. 7 and 17 , at least one hepatic vein was separately draining into right atrium, which were included into Fontan circulation with the conduit involving their ostium. Afterwards, an aortic punch was used to fenestrate the conduit at the posterior side, just above the IVC anastomosis. Then the graft was gently pulled outside the atrium in order to provide a straight and unkinked part to be secured inside the atrial cavity. The right atrial incision was closed with continous polypropylene suture that will enwrap the conduit at the point where it will be directed out to the pericardial cavity. The pulmonary arterial anastomosis of the graft was performed with polypropylene or PTFE sutures. In cases where a pulmonary arterial augmentation was deemed necessary, a bovine pericardial patch (pt. no 12) or a PTFE patch (pt. no 15) was used. There was no chance to prepare autologous pericardium for this purpose due 
to mediastinal adhesions in these redo cases. Total circulatory arrest was not used in any case. In patient 12, a MAPCA was occluded with percutaneous intervention one day before the operation. This patient was the only case where a cavopulmonary anastomosis and IECF were performed at the same stage. Temporary inotropic agents were infused in pt. no 12,13 and 17. Permanent pace maker implantation was not deemed mandatory in any case.

The mean cardiopulmonary bypass and aortic cross clamp times were $93.1+-34.2$ and $70.2+-23.9$ minutes, respectively. All of the patients were extubated after $7.2+-2.6$ hours at the intensive care unit (ICU). The mean ICU stay was $1.7+-0.9$ days. The chest tubes were kept in place until the daily drainage was less than $2 \mathrm{cc} / \mathrm{kg}$. Mean duration of drainage was $5.4+-2.3$ days. Oral warfarin and aspirin were routinely administered in all of the patients. Steroids were administered when the daily drainage exceeded $5 \mathrm{cc} / \mathrm{kg}$ after 5 days of follow up. The target prothrombin time (international normalized ratio, INR) was 2 to 2.5 . We did not make any second intervention for accumulation of pleural fluid after the tubes were removed, except for patient no.15, whom we had to insert pericardial and pleural drainage tubes via subxiphoid approach 13 days after IECF. We did not encounter any phrenic nerve paralysis in this patient population.

Mean follow up period was $3.6+-1.9$ years (range: 1 to 7 years). The mean systemic arterial saturation level at room air was $91.3+-2.7 \%$ (range: $89 \%$ to $96 \%$ ) at the follow up. In pt. no 11, fenestration was closed via femoral access one year after the IECF. Except this case, all of the fenestrations are functioning during the follow-up period, providing a right to left shunt with $5.2+-1.3 \mathrm{mmHg}$ shunt gradient calculated at transthoracic echocardiography. The patients were discharged with diuretics and angiotensin converting enzyme inhibitors (when necessary) in order to achieve systemic decongestion and normal blood pressure.

Fontan failure, associated clinical scenarios (plastic bronchitis or protein losing enteropathy) and mortality was not encountered in our patient population.

\section{DISCUSSION}

Fontan circulation is inherently a non-physiological status where the pulmonary artery trust, i.e., the subpulmonic ventricle is lacking. There is a continous systemic venous congestion secondary to pulmonary vascular resistance and low cardiac output due to decreased filling pressures(11). In the biventricular physiology, the subpulmonic ventricle provides a forward flow to the lungs and the central venous pressure is kept around 2 to $6 \mathrm{mmHg}$. Under such circumstances, the negative intrathoracic pressure and peripheral muscular contractions are not mandatory for providing this pulmonary flow. However, since the forward flow augmentation to the lungs is lacking in Fontan circulation, the systemic venous pressure is increased approximately by three folds (11). In his extraordinary paper, Rychik defined this situation as the 'Fontan paradox' where the high systemic venous pressure is indispensable for adequate cardiac output, and therefore an ideal Fontan may be defined as a failing Fontan as well: a good systemic cardiac output can only be achieved with high systemic venous pressures and congestion (12). The basic pathophysiology of the long-term untoward effects of Fontan circulation is based on these two factors; upstream congestion and downstream decreased flow (13).

Fenestration is one of the most important attempts in order to overcome this viscous cycle (13). In his original report, Dr. Bridges defined the three main advantages of a right to left shunt in Fontan patients; (i) the cardiac output is maintained in expense of systemic desaturation where the pulmonary blood flow is limited; (ii) the uncontrolled increases in systemic venous pressure is eliminated that leads to decreased duration of pleural effusions and (iii) ease of transcatheter closure when necessary (14). In a meta-analysis reported by Dongxu and colleagues, a total of 1929 Fontan patients derived from the data of 14 studies were evaluated (15). Although the postoperative systemic oxygen saturation levels were low in FP with fenestration, the late postoperative oxygen saturation levels did not show any difference statistically. In the same paper, incidence of arrhythmias was found to be lower in fenestrated cases, as well. We did not encounter any arrhythmia in our patient population with the mean follow-up period of 3.6 years. In patients with fenestration of the Fontan pathway, protein losing enteropathy is reported to be less and the thromboembolic complications are not statistically different when compared to non-fenestrated cases $(16,17)$. We did not encounter such 
complications during the follow-up period.

At this point, the question of routine fenestration in FP is still debated in literature. Some centers advocate routine fenestration in order to decrease Fontan failure rates as well as the postoperative pleural effusion (18). However, some papers report worse or similar early outcomes in fenestrated patients $(19,20)$. A recent meta-analysis in a total of 4806 patients sought for the statistical evidence for routine fenestration, however a general consensus was not agreed on (21). Probably the most important result of this analysis was the lack of any scientific evidence that fenestration is associated with lower incidence of Fontan failure. The duration of pleural effusion, MPAP and systemic oxygen saturation levels were lower in fenestrated patients, however the incidence of stroke, duration of hospital stay and early mortality were similar (21).

In our opinion, another advantage of the fenestration in IECF is its durability. All of the fenestrations in our IECF cases are functioning in the follow up period, except one case, which was closed via percutaneous route at another congenital heart disease center. In the era of LT and ECF, the spontaneous closure of surgically created fenestrations could be as high as 19\% (14). Sinha et al. reported that only $66 \%$ of fenestrations were patent at the time of discharge in ECF patients, whereas this ratio was $95 \%$ in IECF patients (22). In a catheter-based analysis in 35 patients, Hirofumi and colleagues reported that persistent fenestration in Fontan circulation suppresses heart rate and CVP, which secures preload reserve without compromising systemic oxygen delivery (23). The high patency rate of IECF may be attributed to more deliberate use of anticoagulant or antiplatelet medications (21). Antiplatelet versus anticoagulation therapy after FP is another matter of debate and anticoagulation combined with antiplatelet drugs seem to be safe with similar incidence of thromboembolic events in Fontan patients (24). We prefer to use combined anticoagulation with antiplatelet treatment in our IECF patients. Recent papers report the promising efficacy and safety of non-vitamin $\mathrm{K}$ antagonist oral anticoagulants at least in adult patients with FP (25).

Another important feature of IECF is decreased incidence of arrhythmias in long-term follow-up (26). Rhythm disturbances in FP patients may lead to decreased cardiac output, AV valve regurgitation, thrombus formation in cardiac chambers and syncope (27). The lateral tunnel modification is traditionally known to cause atrial arrhythmias. However, the ECF technique, which was advocated in the early 1990's as a solution for rhythm disturbances in patients with LT is not innocent as well (26). Transection of IVC and suturing the atrial cuff near terminal crest/Eustachian valve in ECF modification is speculated to damage the atrial conduction pathways and lead to arrhythmia in long term (26). Therefore, preservation of terminal crest and Eustachian valve and eliminating the sutures at the atrial cuff makes the IECF technique superior to LT and ECF with regard to incidence of arrhythmia $(27,28)$. The suture lines in IECF are far from disturbing the conduction pathways and atrium-IVC continuity, as well. Moreover, high patency rates of the fenestration provide a percutaneous access route for intervening any rhythm disturbance if encountered. In our patient population, we did not have any patient with arrhythmia in the postoperative follow-up.

Since an important percentage of the systemic blood flow coming through IVC is directed to pulmonary vascular bed in conduit-based Fontan modifications, the improved hemodynamic performance and minimized energy losses are mandatory for satisfactory long-term results. The conduit in IECF directs the IVC blood in a straight path to the pulmonary arteries, minimizing the energy loss (29). The risk of compression of the pulmonary veins by the conduit, which may be an important problem in ECF is eliminated in IECF (30). The path of the conduit is far away from sinoatrial node and the right atriotomy is more limited, which may have additive effect on decreased incidence of arrhythmias in IECF, as well (26).

Patients undergoing FP with the diagnosis of heterotaxy syndromes is always a challenge and results of FP in these patients are variable in literature $(31,32)$. The IECF modification is easy and reproducible in these patients no matter the anatomy is complex or not, since the cardiopulmonary bypass and aortic cross clamp times are not prolonged with IECF in these patients (27). Another advantage of IECF technique is its reproducibility in patients with separate hepatic venous drainage to the atrium. When ECF is preferred in such patients, the distance between the IVC and hepatic vein ostium as well as the position of the vertebrae should be considered (33). Moreover, it's easy to incorporate the hepatic vein ostium into the IVC-conduit anastomosis within the atrium in IECF technique. We did not encounter any heterotaxy syndrome in our 
patient population, but we had two cases of separate hepatic vein drainage. In their recent series, Hirose K and colleagues recommend to perform IECF in cases with apicocaval juxtaposition (i.e., ipsilateral position of the cardiac apex and IVC) and separated hepatic venous drainage, since the ordinary ECF conduit may be long and too curved that could result in kinking and unbalanced pulmonary blood flow (34).

Two other important parameters when performing a FP are the mean pulmonary artery pressure and pulmonary artery size. Our group previously reported encouraging results of the patients undergoing FP with preoperative MPAP over $15 \mathrm{mmHg}$ (35). This experience was important, since two parameters were still being strict in the traditional 'ten commandments': preoperatively impaired ventricular function and elevated pulmonary artery pressure $(36,37)$. In our patient population, the mean pulmonary artery pressure value was $13.8+-3.3 \mathrm{mmHg}$ which decreased to a mean of $10+-2.4 \mathrm{mmHg}$ after IECF with routine fenestration. On the other hand, the mean pulmonary artery (Nakata) index was $209+-67 \mathrm{~mm}^{2} / \mathrm{m}^{2}$ in our cases, which was above the lower limit of $200 \mathrm{~mm}^{2} / \mathrm{m}^{2}$ stated by paper of Dr. Fontan himself (38). However, much lower limits of $110 \mathrm{~mm}^{2} / \mathrm{m}^{2}$ were emphasized by other authors in literature before a FP is planned (39).

Fontan procedure undoubtfully improved the life expectancy for infants born with complex heart diseases over the last 50 years, however, the morbidity is still high (40). The estimated number of alive Fontan patients only in Europe is over 25.000 (40). The IECF technique which originally combined the advantages of LT and ECF modifications has significant advantages in selected cases. The IECF procedure has the advantage of decreased energy loss through the conduit, since a straight and short pathway is provided with a better position of the graft. The expected incidence of postoperative arrhythmia is lower due to the preserved right atrium - IVC continuity and more limited right atriotomy when compared to ECF. Moreover, fenestration is easy, more durable and easier to be closed via percutaneous route if needed (34).

In our limited patient population, we think that fenestrated intra-extracardiac Fontan procedure may be performed with satisfactory mid-term results both in patients with anatomical complexity or selected patients where the lateral tunnel and extracardiac modifications are not preferred. We prefer IECF especially in patients with complex anatomy such as heterotaxy syndromes and apicocaval juxtaposition. Secondly, in cases where a fenestration is deemed mandatory at the preoperative planning, we prefer this technique, both with regard to technical ease of fenestration as well as its durability. On the other hand, long-term results of this modification are less frequently documented in literature, therefore more studies with larger patient series should be conducted.

\section{ACKNOWLEDGEMENTS}

None

\section{DATA AVAILABILTY STATEMENT}

Data available on request due to privacy/ethical restrictions.

\section{TABLE LEGENDS}

Table - 1: Summary of the patients who underwent Intra-Extracardiac Fontan Procedure.

Table - 2: Details of staged univentricular palliation of the patients who underwent IECF procedure.

\section{AUTHOR CONTRIBUTIONS}

BS: Drafting the article, data analysis/interpretation, data collection.

AO: Concept/design, data analysis/interpretation, critical revision of the article.

ŞD: Data analysis/interpretation, data collection.

MS: Data collection, drafting the article.

MSB: Concept/design, critical revision of the article, approval of the article.

\section{REFERENCES}


1. Fontan F, Baudet E. Surgical repair of tricuspid atresia. Thorax 1971;26(3):240-248.

2. Jonas RA. The intra/extracardiac conduit fenestrated Fontan. Semin Thorac Cardiovasc Surg Pediatr Card Surg Annu 2011;14(1):11-18.

3. Jonas RA, Castenada AR. Modified Fontan procedure: atrial baffle and systemic venous to pulmonary artery anastomotic techniques. J Card Surg 1988;3(2):91-96.

4. de Leval MT, Kilner P, Gewillig M et al. Total cavopulmonary connection: a logical alternative to atriopulmonary connection for complex Fontan operations. Experimental studies and early clinical experience. J Thorac Cardiovasc Surg 1988;96:682-695.

5. Marceletti C, Corno A, Giannico S et al. Inferior vena cava-pulmonary artery extracardiac conduit. A new form of right heart bypass. J Thorac Cardiovasc Surg 1990:100:228-232.

6. Rodefeld MD, Bromberg BI, Schuessler RB et al. Atrial flutter after lateral tunnel construction in the modified Fontan operation: a canine model. J Thorac Cardiovasc Surg 1996;111(3):514-526.

7. Bridges ND, Lock JE, Castenada AR. Baffle fenestration with subsequent transcatheter closure. Modification of the Fontan operation for patients at increased risk. Circulation 1990;82:1681-1689.

8. Lemler MS, Scott WA, Leonard SR et al. Fenestration improves clinical outcome of the Fontan procedure: a prospective, randomized study. Circulation 2002;105(2):207-212.

9. Vargas FJ, Mayer JE, Jonas RA, Castenada AR. Anomalous systemic and pulmonary venous connections in conjunction with atriopulmonary anastomosis (Fontan-Kreutzer). Technical considerations. J Thorac Cardiovasc Surg 1987;93(4):523-532.

10. Michielon G, Gharagozloo F, Julsrud PR et al. Modified Fontan operation in the presence of anomalies of systemic and pulmonary venous connection. Circulation 1993;88(II): 141-148.

11. Talwar S, Marathe SP, Choudhary SK, Airan B. Where are we after 50 years of the Fontan operation? J Thorac Cardiovasc Surg 2021;37(suppl 1):42-53.

12. Rychik J. The relentless effects of the Fontan Paradox. Semin Thorac Cardiovasc Surg Pediatr Card Surg Annu 2016;19(1):37-43.

13. Gewellig M, Brown SC. The Fontan circulation after 45 years: update in physiology. Heart 2016;102(14):1081-1086.

14. Bridges ND, Mayer JE, Lock JE et al. Effect of baffle fenestration on outcome of the modified Fontan operation. Circulation 1992;86(6):1762-1769.

15. Li D, Li M, Zhou X, An Q. Comparison of the fenestrated and non-fenestrated Fontan procedures: A meta-analysis. Medicine (Baltimore) 2019;98(29):e16554.

16. Atz AM, Travison TG, McCrindle BW et al. Late status of Fontan patients with persistent surgical fenestration. J Am Coll Cardiol 2011;57(24):2437-2443.

17. Fu S, Valeske K, Akinturk H, Schranz D. Fontan extracardiac tunnel connection: fenestration or not? Chin Med J (Engl) 2009;122(19):2335-2338.

18. Airan B, Sharma R, Choudhary SK et al. Univentricular repair: is routine fenestration justified? Ann Thorac Surg 2000;69(6):1900-1906.

19. Fan F, Wang Q, Yi T et al. Impact of fenestration on early outcome in patients undergoing modified Fontan operation. Zhonghua Xin Xue Guan Bing Za Zhi 2015;43(6):527-530.

20. Fan F, Liu Z, Shoujun L et al. Effect of Fenestration on Early Postoperative Outcome in Extracardiac Fontan Patients with Different Risk Levels. Pediatr Cardiol 2017;38(4):643-649. 
21. Bouhout I, Walid BA, Khalaf D, Raboisson MJ, Poirier N. Effect of Fenestration on Fontan Procedure Outcomes: A Meta-Analysis and Review. Ann Thorac Surg 2020;109(5):1467-1474.

22. Sinha L, Ozturk M, Zurakowski D et al. Intra-Extracardiac Versus Extracardiac Fontan Modifications: Comparison of Early Outcomes. Ann Thorac Surg 2019;107(2):560-566.

23. Saiki H, Kuwata S, Iwamoto Y et al. Fenestration in the Fontan circulation as a strategy for chronic cardioprotection. Heart 2019;105(16):1266-1272.

24. Marrone C, Galasso G, Piccolo R et al. Antiplatelet versus anticoagulation therapy after extracardiac conduit Fontan: a systematic review and meta-analysis. Pediatr Cardiol 2011;32(1):32-39.

25. Yang H, Gruschen R, Veldtman R et al. Non-vitamin K antagonist oral anticoagulants in adults with a Fontan circulation: are they safe. Heart 2019;6(1):e000985.

26. Talwar S, Sengupta S, Choudhary SK. The intra-extracardiac Fontan: preliminary results

Indian J Thorac Cardiovasc Surg 2020;36(3):193-198.

27. Fishberger SB, Wernovsky G, Gentles TL et al. Factors that influence the development of atrial flutter after the Fontan operation. J Thorac Cardiovasc Surg. 1997;113(1):80-86.

28. 28. Sinha P, Zurakowski D, He D et. al. Intra/extracardiac fenestrated modification leads to lower incidence of arrhythmias after the Fontan operation. J Thorac Cardiovasc Surg 2013;145(3):678-682.

29. Itatani K, Miyaji K, Tomoyasu T et al. Optimal conduit size of the extracardiac Fontan operation based on energy loss and flow stagnation. Ann Thorac Surg. 2009;88(2):565-572; discussion 572-573.

30. Kugo Y, Iwai S, Ishimaru K et al. Pulmonary venous obstruction after extracardiac total cavopulmonary connection in right atrial isomerism. Gen Thorac Cardiovasc Surg 2020;68(9):969-974.

31. Marathe SP, Zannino D, Cao JY et al. Heterotaxy Is Not a Risk Factor for Adverse Long-Term Outcomes After Fontan Completion. Ann Thorac Surg 2020;110(2):646-653.

32. Marathe SP, Jacob YC, Celermajer D et al. Outcomes of the Fontan Operation for Patients With Heterotaxy: A Meta-Analysis of 848 Patients. Ann Thorac Surg 2020;110(1):307-315.

33. Nakata T, Fujimoto Y, Hirose K et al. Fontan completion in patients with atrial isomerism and separate hepatic venous drainage. J Cardiothorac Surg 2010;37(6):1264-1270.

34. Hirose K, Ikai A, Murata M et al. Intra-Extracardiac Total Cavopulmonary Connection for Patients With Anatomical Complexity. Ann Thorac Surg 2021;111(3):958-965.

35. Özyüksel A, Şimşek B, Özden Ö et al. Fontan procedure in patients with preoperative mean pulmonary artery pressure over $15 \mathrm{mmHg}$. J Card Surg 2021;36(3):941-949.

36. Choussat A, Fontan F, Besse P. Selection criteria for the Fontan procedure. In: Anderson RH, Shinebourne (editors) Pediatric Cardiology. Churchill Livingstone, Edinburgh, Scotland (1977) pp:559-566.

37. Hosein RBM, Clarke AJB, Mc Guirk SP et al. Factors influencing early and late outcome following the Fontan procedure in the current era. The 'Two Commandments'? Eur J Cardiothorac Surg 2007;31(3):344352.

38. Fontan F, Fernandez G, Costa F et al. The size of the pulmonary arteries and the results of the Fontan operation. J Thorac Cardiovasc Surg. 1989;98(5 Pt 1):711-719; discussion 719-724.

39. Itatani K, Miyaji K, Nakahata Y et al. The lower limit of the pulmonary artery index for the extracardiac Fontan circulation. J Thorac Cardiovasc Surg. 2011;142(1):127-135.

40. Hedlund E, Lundell B. Fontan circulation has improved life expectancy for infants born with complex heart disease over the last 50 years but has also resulted in significant morbidity. Acta Paediatr 00:1-6. 


\section{Hosted file}

IECF - Table-1.docx available at https://authorea.com/users/354960/articles/547302-mid-termresults-of-fenestrated-intra-extracardiac-fontan-procedure-insights-experiences-andexpectations

\section{Hosted file}

IECF - Table-2.docx available at https://authorea.com/users/354960/articles/547302-mid-termresults-of-fenestrated-intra-extracardiac-fontan-procedure-insights-experiences-andexpectations 